\title{
Frequency of microbial isolates and pattern of antimicrobial resistance in patients with hematological malignancies: a cross-sectional study from Palestine
}

Genan Arman ${ }^{1}$, Marwa Zeyad', Beesan Qindah', Adham Abu Taha ${ }^{2,3}$, Riad Amer ${ }^{1,4}$, Shatha Abutaha', Amer A. Koni $i^{5,6}$ and Sa'ed H. Zyoud ${ }^{5,7,8,9^{*}}$ (1)

\begin{abstract}
Background: Infections are the main cause of death in patients with hematologic malignancies. This study aims to determine the microbial profile of infections in patients with hematologic malignancies and to determine the antimicrobial resistance patterns for these pathogens.

Methods: A retrospective descriptive cross-sectional study was conducted from January 2018 to December 2019 at a large hematological center in Palestine. The medical data of hematologic malignancy patients with positive cultures were collected from the hematology/oncology department using the hospital information system, and data regarding the microbial isolates and their antimicrobial resistance were collected from the microbiology laboratory.

Results: A total of 144 isolates were identified from different types of specimens, mostly blood samples. Of all isolates, 66 (45.8\%) were gram-negative bacteria (GNB), 57 (39.6\%) were gram-positive bacteria (GPB), and 21 (14.6\%) were fungal isolates. The GNB that were most frequently isolated were Pseudomonas aeruginosa (27, 40.9\%), followed by Escherichia coli (E. coli) (20,30.3\%). Fourteen isolates (24.6\%) of GPB were Staphylococcus epidermidis followed by Enterococcus faecium $(10,17.5 \%)$ and Staphylococcus hemolyticus (10, 17.5\%). The most frequent fungal pathogens were Candida species (20,95.2\%). GNB were found to be resistant to most antibiotics, mainly ampicillin (79.3\%). Pseudomonas aeruginosa exhibited high resistance to ciprofloxacin (60\%) and imipenem (59.3\%). Among GPB, high resistance rates to oxacillin (91.1\%) and amikacin (88.8\%) were found. All isolated strains of Staphylococcus epidermidis were resistant to cephalosporins and oxacillin. Approximately half of the GNB isolates (34,51.5\%) were multi-drug resistant organisms (MDRO), and 16.7\% (11 isolates) were difficult-to-treat resistance (DTR). Furthermore, 68.4\% (39 isolates) of GPB were MDRO. The proportion of staphylococci (CoNS and S. aureus) resistant to oxacillin was $91.7 \%$, while $88.6 \%$ of enterococci were resistant to vancomycin.
\end{abstract}

Conclusions: The findings of this study confirm the predominant microorganisms seen in patients with hematologic malignancies, and show a high percentage of antibiotic resistance. Policies regarding antibiotic use and proper

*Correspondence: saedzyoud@yahoo.com; saedzyoud@najah.edu

${ }^{5}$ Department of Clinical and Community Pharmacy, College of Medicine

and Health Sciences, An-Najah National University, Nablus 44839,

Palestine

Full list of author information is available at the end of the article

(C) The Author(s) 2022. Open Access This article is licensed under a Creative Commons Attribution 4.0 International License, which permits use, sharing, adaptation, distribution and reproduction in any medium or format, as long as you give appropriate credit to the original author(s) and the source, provide a link to the Creative Commons licence, and indicate if changes were made. The images or other third party material in this article are included in the article's Creative Commons licence, unless indicated otherwise in a credit line to the material. If material is not included in the article's Creative Commons licence and your intended use is not permitted by statutory regulation or exceeds the permitted use, you will need to obtain permission directly from the copyright holder. To view a copy of this licence, visit http://creativecommons.org/licenses/by/4.0/. The Creative Commons Public Domain Dedication waiver (http://creativeco mmons.org/publicdomain/zero/1.0/) applies to the data made available in this article, unless otherwise stated in a credit line to the data. 
infection control measures are needed to avert the ever-growing danger of antimicrobial resistance. This may be achieved by developing antibiotic stewardship programs and local guidelines based on the hospital's antibiogram.

Keywords: Bacterial isolates, Antimicrobial resistance, Hematologic malignancies, Palestine

\section{Background}

In recent decades, major developments have been made in the care of cancer patients that have significantly improved patient survival. However, despite these developments, patients with hematologic malignancies remain at an extraordinarily high risk of infections. This is the result of a complex interaction between basic immunodeficiency and therapeutic practices such as surgery, radiation, and chemotherapy [1-4].

Previous studies have reported that the prevalence of bacterial bloodstream infections among patients with hematologic malignancies ranges from 11 to $38 \%$, and the rough mortality rate reaches up to 40\% [5-7]. Furthermore, studies have shown gram-negative bacteria (GNB) to be the most frequently isolated organisms during the 1960s and 1970s [8]. However, over the following two decades, the proportion of gram-positive bacteria (GPB) has increased.

A study in India showed that E. coli was the most common isolated organism, followed by coagulase-negative staphylococci (CoNS) [9]. Another study showed that GNB were the most common isolated organisms, and the best empirical treatment for them was non-carbapenembased anti-pseudomonal antibiotics [5]. However, another study conducted in 2018 in Ethiopia showed a change in prevalence from GNB to GPB, mainly as a result of increased use of urinary catheters, and multi-drug resistance was detected in $46.3 \%$ of bacterial isolates [10].

In Palestine, cancer is the third leading cause of death after heart disease and cerebrovascular disease, accounting for $10.3 \%$ of total deaths [11]. However, Palestinian cancer services started to spring up in the early 2000s, and it took until 2008 to organize cancer care at Augusta Victoria Hospital in East Jerusalem [12]. In the Gaza Strip, a study showed a high prevalence of resistance to amoxicillin (73.2\%) in isolates of Staphylococcus aureus, and high resistance to penicillin (40.4\%) in Streptococcus pneumonia [13]. However, no published reports have shown the epidemiology and antimicrobial resistance patterns of potential etiological agents among patients with hematologic malignancies in Palestine.

Antibiotic resistance is a growing concern in global health [14-20]. Overuse and constant consumption of antibiotics, due to lack of control programs in hospitals and over-the-counter antibiotics, lead to multi-drug resistant pathogens. These then lead to increased mortality, length of hospitalizations, and health care costs [21, 22].
This study provides information on the spectrum of microbial isolates and their antimicrobial resistance patterns in patients with hematologic cancer at An-Najah National University Hospital. This study is the first to evaluate bacterial and fungal resistance patterns among hematological malignancies in Palestine. This information will help decrease morbidity and mortality by helping to establish empirical treatment guidelines and antibiotic stewardship programs. These will reduce antibiotic overuse and, subsequently, decrease hospitalizations. This study also highlights the immense effect and burden of multi-drug resistant organisms.

\section{Methods}

Study design

A retrospective cross-sectional study was conducted to determine the frequency of microbial isolates and patterns of antimicrobial resistance in patients with hematologic malignancies.

\section{Study setting}

Data were collected from the medical laboratory department and the hematology department of An-Najah National University Hospital. The hospital has a bed capacity of 169 , with approximately 40 beds for adult hematology/oncology patients [23].

\section{Study population}

All patients with hematologic malignancies who had a positive culture from January 2018 to December 2019 at An-Najah National University Hospital.

\section{Data collection}

Demographic and medical data were obtained from the hospital information system. Information on the sources of specimens, types of microorganisms, and antibiotic susceptibility was collected from the microbiology laboratory. Importantly, cefepime is available in the hospital, but its use is very restricted because it requires a special request from an infectious disease specialist. Furthermore, ceftolozane / tazobactam is not available in Palestine. 


\section{Inclusion criteria}

All patients with hematologic malignancies who had positive cultures during the study period at An-Najah National University Hospital were included.

\section{Exclusion criteria}

Patients who did not have a positive culture and those who had solid tumors were excluded.

\section{Ethical considerations}

The proposal was reviewed and accepted on September 22, 2019, with the permission of An-Najah National University Hospital. The approval of the Institutional Review Board (IRB) Committee of An-Najah National University was obtained on July 24, 2019 (Archived number: AN4June2019).

\section{Statistical analysis}

Data were entered and analyzed using version 21 of the Statistical Package for Social Sciences (SPSS) program. For continuous variables, data were expressed as means \pm standard deviation (SD) and as frequencies and percentages for categorical variables.

\section{Results}

\section{Demographic and clinical characteristics of the study population}

A total of 144 cancer patients were included in the study. Of these, 77 (53.5\%) were women and 67 (46.5\%) were men. The mean age \pm SD of the study participants was $40.8 \pm 16.6$ years, ranging from 17 to 84 years old. The most common hematologic malignancy was acute lymphoid leukemia (ALL) (36, 25\%), followed by Hodgkin's lymphoma (33, 22.9\%), acute myeloid leukemia $(30,20.8 \%)$, multiple myeloma $(22,15.3)$, non-Hodgkin lymphoma $(16,11.1 \%)$, chronic lymphoid leukemia (4, $2.8 \%)$, Waldenstrom macroglobulinemia (2, 1.4\%) and Langerhans cell histiocytosis (1, 0.7\%). Most of these patients were actively on the chemotherapy protocol $(83,57.6 \%)$ or had had bone marrow transplantation $(35,24.3 \%)$, while others had finished their treatment $(17,11.8 \%)$ or had not received any cancer treatment at the time their samples were collected $(9,6.3 \%)$. It should be noted that most of the patients were febrile $(91,63.2 \%)$ and a portion had died at the end of the study (12, 8.3\%); (Table 1).

\section{Microbial profiles and site of isolation}

A total of 144 microbial samples were collected, the majority isolated from blood $(57,39.6 \%)$, urine (40, $27.8 \%)$ and sputum $(17,11.8 \%)$. A smaller portion was isolated from fluid (3, 2.1\%; one from ascetic fluid and
Table 1 Demographic and clinical characteristics of cancer patients with positive cultures

\begin{tabular}{lll}
\hline Variable & & $\mathbf{n}(\%)$ \\
\hline Age(years), mean \pm SD & & $40.8 \pm 16.6$ \\
Sex & Male & $67(46.5)$ \\
Type of cancer & Female & $77(53.5)$ \\
& HLL & $36(25)$ \\
& AML & $33(22.9)$ \\
& MM & $30(20.8)$ \\
& Non-Hodgkin's lymphoma & $22(15.3)$ \\
& CLL & $16(11.1)$ \\
& Waldenstrom macroglobulinemia & $2(1.4)$ \\
Cancer treatments & Langerhans cell histiocytosis & $1(0.7)$ \\
& Chemotherapy & $83(57.6)$ \\
& Bone Marrow Transplant & $35(24.3)$ \\
& Completed treatment & $17(11.8)$ \\
& No treatment & $9(6.3)$ \\
Febrile & Yes & $91(63.2)$ \\
& No & $53(36.8)$ \\
& Yes & $12(8.3)$ \\
& No & $132(91.7)$ \\
\hline
\end{tabular}

ALL acute lymphoid leukemia, $A M L$ acute myeloid leukemia, $M M$ multiple myeloma, CLL chronic lymphoid leukemia

the other two unspecified), stool $(2,1.4 \%)$ and cerebrospinal fluid (1, 0.7\%).

There were 144 positive cultures; 66 (45.8\%) isolates were GNB, 57 (39.6\%) GPB, and fungal infections were positive in $21(14.6 \%)$ samples.

Among GNB, Pseudomonas aeruginosa (27, 40.9\%) was predominant, followed by E. coli $(20,30.3 \%)$ which can be divided into non-extended-spectrum beta-lactamase-producing Escherichia coli (non-ESBL-EC); (10, 50\%), and ESBL-producing Escherichia coli (ESBLEC); (10, 50\%). These were followed by both Acinetobacter baumannii and Klebsiella pneumonia (6, 9.1\%) (Table 2).

In terms of GPB, Staphylococcus epidermidis represented the most frequent species $(14,24.6 \%)$, followed by Enterococcus faecium and Staphylococcus hemolyticus (both 10, 17.5\%), Staphylococcus hominis (6, 10.5\%), Enterococcus faecalis (5, 8.7\%), and Staphylococcus aureus (4, 7.0\%) (Table 2).

Fungal infections were positive in 21 samples (14.6\%). The vast majority were Candida spp. (20,95.2\%) with only one (4.8\%) Saprochaete capitate (Table 2). Among the 20 isolated Candida spp., eight were Candida tropicalis, seven were Candida glabrata, four were Candida albicans, and one was Candida dubliniensis. 
Table 2 Frequency and percentage of isolated microorganisms

\begin{tabular}{|c|c|}
\hline Microorganism & Frequency \\
\hline Total number 144 & $N(\%)$ \\
\hline Gram-negative, total & $66(45.8)$ \\
\hline Pseudomonas aeruginosa & $27(40.9)$ \\
\hline E. coli & $20(30.3)$ \\
\hline Non-ESBL & $10(50)$ \\
\hline ESBL & $10(50)$ \\
\hline Acinetobacter baumannii & $6(9.1)$ \\
\hline Klebsiella pneumonia & $6(9.1)$ \\
\hline Enterobacter cloacae & $2(3.2)$ \\
\hline Salmonella species & $1(1.5)$ \\
\hline Sphingomonas paucimobilis & $1(1.5)$ \\
\hline Shigella species & $1(1.5)$ \\
\hline Raoultella planticola & $1(1.5)$ \\
\hline Haemophilus influenzae & $1(1.5)$ \\
\hline Gram-positive, total & $57(39.6)$ \\
\hline Staphylococcus epidermidis & $14(24.6)$ \\
\hline Enterococcus faecium & $10(17.5)$ \\
\hline Staphylococcus haemolyticus & $10(17.5)$ \\
\hline Staphylococcus hominis & $6(10.5)$ \\
\hline Enterococcus faecalis & $5(8.7)$ \\
\hline Staphylococcus aureus & $4(7.0)$ \\
\hline Streptococcus agalactia & $2(3.5)$ \\
\hline Micrococcus luteus & $2(3.5)$ \\
\hline Streptococcus oralis & $1(1.8)$ \\
\hline Staphylococcus capitis & $1(1.8)$ \\
\hline Staphylococcus sciuri & $1(1.8)$ \\
\hline Kocuria varians & $1(1.8)$ \\
\hline Fungal infection & $21(14.6)$ \\
\hline Candida species & $20(95.2)$ \\
\hline Candida tropicalis & $8(38.1)$ \\
\hline Candida glabrata & $7(33.3)$ \\
\hline Candida albicans & $4(19)$ \\
\hline Candida dubliniensis & $1(4.8)$ \\
\hline Saprochaete capitate & $1(4.8)$ \\
\hline
\end{tabular}

E. coli Escherichia coli, ESBL extended-spectrum beta-lactamase

\section{Antimicrobials used before and after culture}

The vast majority of antimicrobials were used in combinations. Among those used in combination, amikacin was the most popular antibiotic for empiric therapy, since it was used in 45 cases out of 144 organisms $(31.3 \%, 45 / 144)$, followed by vancomycin $(30.5 \%$, 44/144), meropenem $(27.8 \%, 40 / 144)$, piperacillintazobactam $(27.8 \%, 40 / 144)$, colistin $(23.6 \%, 34 / 144)$ and tigecycline $(18.6 \%, 27 / 144)$. On the other hand, the least frequently used antimicrobials were azithromycin, clindamycin, and caspofungin $(0.7,1 / 144)$ (Table 3$)$.

For culture-guided antibiotic use, the antibiotic most frequently used was meropenem (41.7\%, 60/144),
Table 3 Frequency and percentage of empiric antimicrobials (before the results of the culture)

\begin{tabular}{|c|c|}
\hline Empiric antimicrobials & $\begin{array}{l}\text { Frequency } \\
\mathrm{N}(\%)\end{array}$ \\
\hline Amikacin* & $45(31.3)$ \\
\hline Vancomycin* & $44(30.3)$ \\
\hline Meropenem* & $40(27.8)$ \\
\hline Meropenem** & $2(1.4)$ \\
\hline Piperacillin-Tazobactam* & $40(27.8)$ \\
\hline Piperacillin-Tazobactam** & $1(0.7)$ \\
\hline Colistin* & $34(23.6)$ \\
\hline Colistin** & $1(0.7)$ \\
\hline Tigecycline* & 27 (18.6) \\
\hline Voriconazole* & $21(14.6)$ \\
\hline Fluconazole* & $18(12.5)$ \\
\hline Miconazole* & $18(12.5)$ \\
\hline Levofloxacin* & $16(11.1)$ \\
\hline Levofloxacin** & $1(0.7)$ \\
\hline Trimethoprim-Sulfamethoxazole* & $15(10.4)$ \\
\hline Ceftazidime* $^{*}$ & $10(6.9)$ \\
\hline Acyclovir* & $10(6.9)$ \\
\hline Metronidazole* & $8(5.6)$ \\
\hline Metronidazole ${ }^{* *}$ & $1(0.7)$ \\
\hline Ciprofloxacin* & $6(4.5)$ \\
\hline Ciprofloxacin** & $1(0.7)$ \\
\hline Ceftriaxone* & $4(2.8)$ \\
\hline Azithromycin* & $1(0.7)$ \\
\hline Azithromycin** & $1(0.7)$ \\
\hline Amphotericin* & $2(1.4)$ \\
\hline Caspofungin* & $1(0.7)$ \\
\hline Clindamycin* & $1(0.7)$ \\
\hline
\end{tabular}

"Used in combination therapy, ${ }^{* *}$ used as monotherapy

followed by colistin $(33.3 \%, 48 / 144)$, tigecycline $(26.4 \%$, $38 / 144)$, vancomycin $(25 \%, 36 / 144)$ and amikacin $(22.9 \%$, $33 / 144)$. Meanwhile, the antibiotics used most frequently were linezolid $(0.7 \%, 1 / 144)$, followed by amoxicillin-clavulanate $(1.4 \%, 2 / 144)$ (Table 4$)$. De-escalation of therapy occurred in $52(36.1 \%)$ cases in the sample.

\section{Antimicrobial resistance of gram-negative bacterial isolates}

In general, the highest resistance rates for GNB were $79.3 \%$ for ampicillin, $73.3 \%$ to levofloxacin, $65.7 \%$ for ceftriaxone, $61.8 \%$ to piperacillin, and $59.3 \%, 56.5 \%$ and $59.4 \%$ for cefuroxime, ciprofloxacin, and tobramycin, respectively. Meanwhile, the lowest resistance rates were 3.6\% for ertapenem, $6.25 \%$ for nitrofurantoin, $8.3 \%$ to colistin, and $16.3 \%$ to piperacillin-tazobactam. Among GNB, 23 isolates (34.8\%) were ESBL and 23 isolates (31.8\%) were CRE (carbapenem resistant Enterobacteriaceae). The most common isolated GNB, Pseudomonas aeruginosa, had a high 
Table 4 Frequency and percentage of antimicrobials after the results of the culture

\begin{tabular}{ll}
\hline Antimicrobials & Frequency \\
N (\%)
\end{tabular}

resistance rate to ciprofloxacin (60\%), imipenem (59.3\%), piperacillin (54.2\%), meropenem, and gentamicin (48\% each). Furthermore, resistance rates against cephalosporins, cefepime, and ceftazidime were $16 \%$ and $24 \%$ respectively. E. coli isolates were highly susceptible to amikacin (95\%), while they were highly resistant to trimethoprimsulfamethoxazole and fluoroquinolones. Regarding the six isolates of Acinetobacter baumannii, the highest resistance rates were towards carbapenems (80\% for meropenem and $83.3 \%$ to imipenem), piperacillin-tazobactam $(83.3 \%)$ and gentamicin (66.7\%). Only four isolates were tested for sensitivity to colistin and all showed $0 \%$ resistance. Finally, the six isolates of Klebsiella pneumonia were highly susceptible to piperacillin-tazobactam, carbapenems, fluoroquinolones ceftazidime and cefepime. The antimicrobial resistance profiles of the most frequently isolated GNB are reported in Table 5.

Antimicrobial resistance of gram-positive bacterial isolates Among the CoNS isolated in our study (S. epidermidis, hemolyticus, hominis, sciuri, and capitis( and Staphylococcus aureus, none were resistant to vancomycin or linezolid, while $93.3 \%$ of CoNS and 75\% of Staphylococcus aureus were resistant to oxacillin. Regarding the 14 isolates of Staphylococcus epidermidis isolates, all were resistant to penicillin and cephalosporins and $54.5 \%$ were resistant to trimethoprim-sulfamethoxazole. Among the 10 isolates of Enterococcus faecium and 5 of Enterococcus faecalis, 90\% of Enterococcus faecium isolates were resistant to vancomycin (VRE) while none of the Enterococcus faecalis isolates were VRE. 40\% of Enterococcus faecium isolates were resistant to streptomycin, 30\% were resistant to gentamicin, and $11.1 \%$ were resistant to tigecycline. Meanwhile, Enterococcus faecalis species had $80 \%$ resistance to streptomycin, $50 \%$ resistance to gentamicin, and $33.3 \%$ resistance to tigecycline. However, none of the Enterococcus faecalis or Enterococcus faecium isolates were resistant to linezolid. Overall, the highest resistance rates of GPB were $91.1 \%$ to oxacillin, $88.8 \%$ to amikacin, $86.9 \%$ to cefuroxime, $85.1 \%$ to erythromycin, and $84.8 \%, 82.6 \%, 77.4$ to penicillin, ceftriaxone, amoxicillin-clavulanic, respectively. Meanwhile, the lowest resistance rates were $0 \%$ to linezolid, $4.2 \%$ to tigecycline, $11.5 \%$ to quinupristin-dalfopristin, and $16.1 \%$ to vancomycin. The antimicrobial resistance profiles of the most frequently isolated GPB are reported in Table 6.

\section{Antifungal resistance and sensitive profiles of fungal organisms}

As for antifungals, no resistance was found to caspofungin, fluconazole, flucytosine, voriconazole, or micafungin.

\section{Multi-drug resistant organisms (MDRO)}

MDROs are defined as those resistant to at least one agent in three or more antimicrobial categories [24]. $51.5 \%$ of GNB isolates and $68.4 \%$ of GPB isolates were found to be MDRO. Among GNB, Acinetobacter baumannii had the highest rate of MDRO (83.3\%), whereas among GPB, CoNS had the highest rate (81.3\%) (Table 7).

\section{Difficult-to-treat resistance (DTR) of GNB}

DTR is defined as an isolate demonstrating intermediate or resistant phenotype to all reported agents in carbapenem, $\beta$-lactam, and fluoroquinolone categories, including additional agents, such as piperacillin-tazobactam and ampicillin-sulbactam ( $A$. baumannii only), and aztreonam (not applicable to $A$. baumannii), when results are available [25]. In this study, 11 isolates (16.7\%) were DTR; 5 (18.5\%) were Pseudomonas aeruginosa, 5 (83.3\%) were Acinetobacter baumanni (6) and 1 (5\%) was E. coli (Table 7).

\section{Discussion}

Infections are the most common cause of death in cancer patients, especially among those with hematologic malignancies, with studies reporting that approximately 


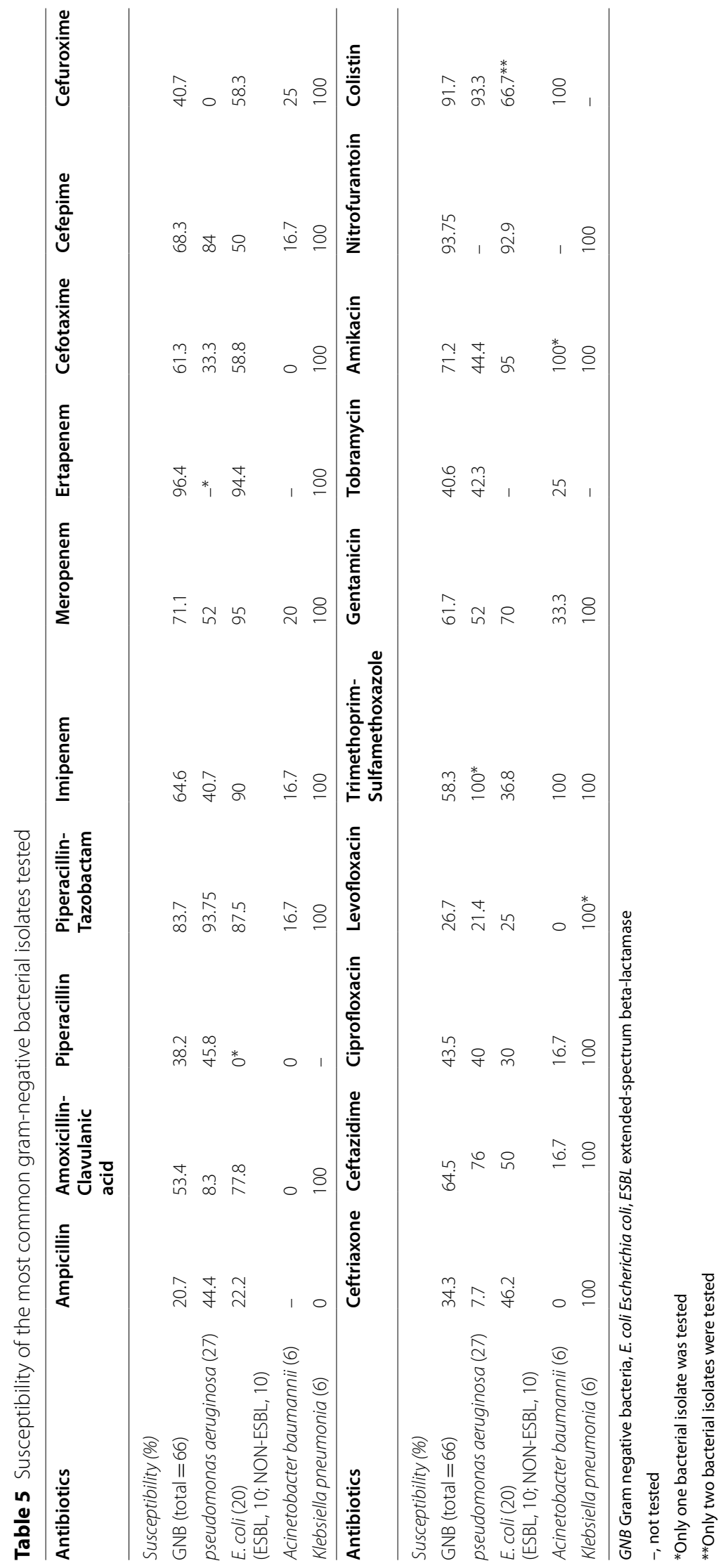




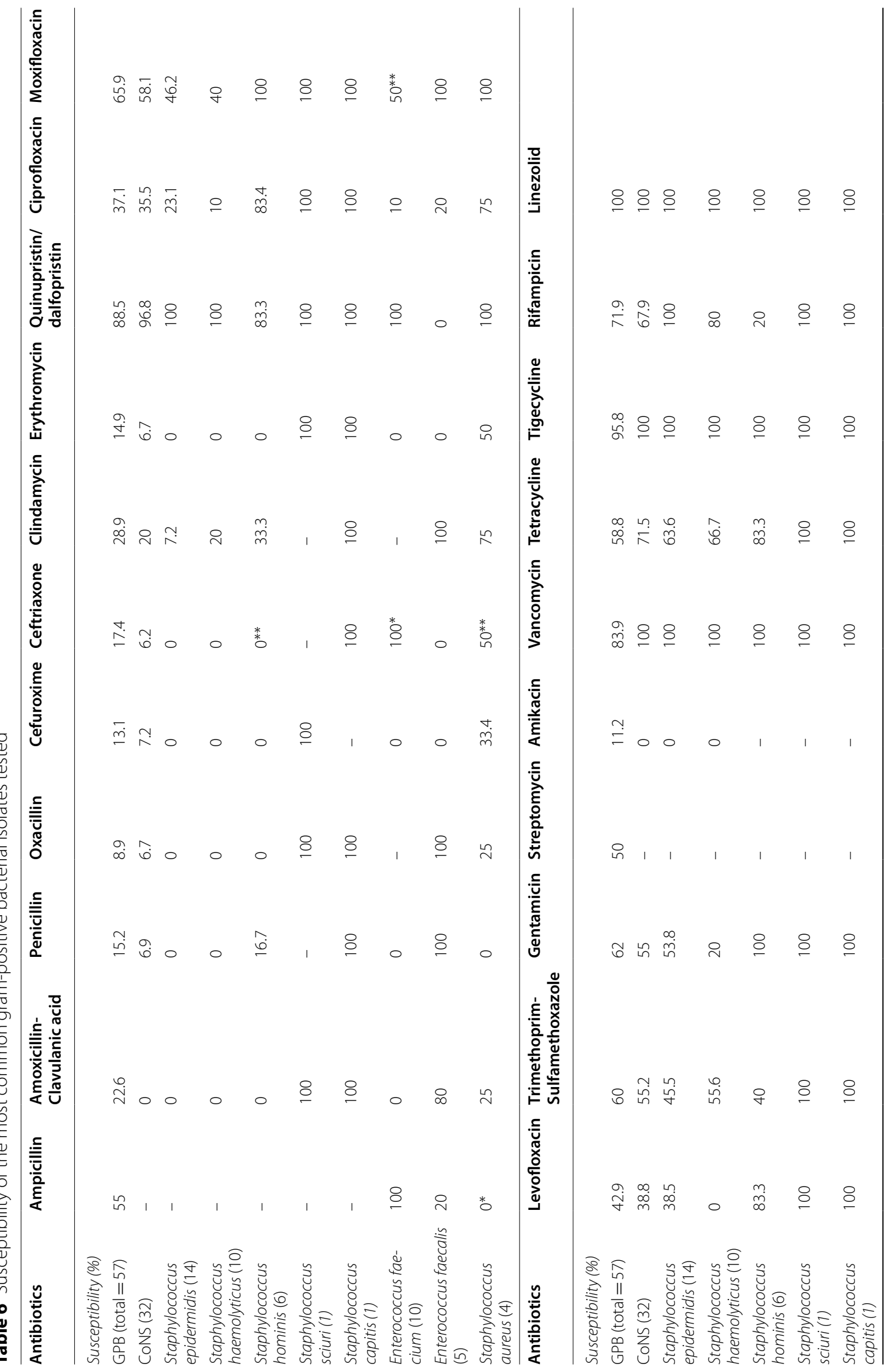




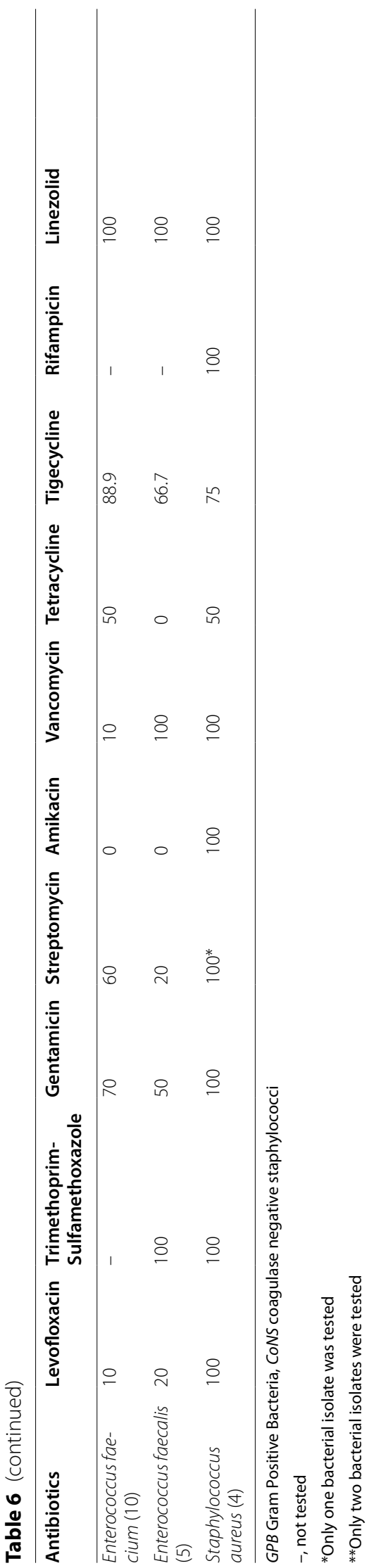


Table 7 Frequency and percentage of multidrug resistant organisms

\begin{tabular}{lll}
\hline Microorganism & $\begin{array}{l}\text { MDRO } \\
\mathbf{n}(\%)\end{array}$ & $\begin{array}{l}\text { DTR } \\
\mathbf{n}(\%)\end{array}$ \\
\hline GNB (66) & $\mathbf{3 4 ( 5 1 . 5 )}$ & $\mathbf{1 1}(\mathbf{1 6 . 7 )}$ \\
Pseudomonas aeruginosa (27) & $15(55.6)$ & $5(18.5)$ \\
E. coli (20) & $13(65)$ & $1(5)$ \\
Acinetobacter baumanni (6) & $5(83.3)$ & $5(83.3)$ \\
Klebsiella pneumonia (6) & $0(0)$ & $0(0)$ \\
Enterobacter spp. (5) & $1(20)$ & $0(0)$ \\
GPB (57) & $\mathbf{3 9 ( 6 8 . 4 )}$ & - \\
CoNS (32) & $26(81.3)$ & - \\
Enterococcus spp. (15) & $11(73.3)$ & - \\
Staphylococcus aureus (4) & $2(50)$ & -
\end{tabular}

Enterobacter spp.: Enterobacter cloacae, Salmonella species, Raoultella planticola, and Shigella species; Enterococcus spp.: Enterococcus faecium and Enterococcus faecalis

MDRO multidrug resistant organisms, GNB Gram-Negative Bacteria, GPB GramPositive Bacteria, E. coli Escherichia coli, spp. Species, CoNS coagulase-negative Staphylococci, DTR difficult-to-treat resistance

$60 \%$ of deaths are infection-related [26]. This increased risk of infections can be due to host or treatment-related causes. Host-related factors consist of immunodeficiency, comorbid illnesses, mucosal ulcerations, previous infections, nutritional deficiency, and stress [26], while treatment-related factors include invasive procedures, surgery, radiation, immunosuppressive drugs, and use of antimicrobials [27]. These infections can be caused by various pathogens such as viruses, bacteria, fungi, etc. Bacteria are the leading cause of infections in cancer patients, followed by fungi [27].

In our study, Pseudomonas aeruginosa (27, 43.6\%) was the predominant bacterium among GNB, followed by $E$. coli $(20,32.3 \%)$ that can be divided into non-ESBL (10, $50 \%)$ and ESBL-E. coli (10, 50\%). These were followed by Acinetobacter baumannii and Klebsiella pneumonia, with six isolates each (9.7\%). These results are in conjunction with other studies conducted in India and Pakistan. In the former, they reconfirmed the predominance of GNB in patients with hematologic cancers, with E. coli, Pseudomonas, and Klebsiella having the largest shares [28] In the latter study, which evaluated GNB isolated from bloodstream infections of patients on chemotherapy, Pseudomonas aeruginosa was the most frequent bacteria, followed by E. coli, Klebsiella, Proteus, and Shigella [29]. These results are also similar to a study conducted in Italy, where E. coli was the most frequent organism, followed by Pseudomonas aeruginosa, Klebsiella pneumoniae, and Enterobacter cloacae [30]. In another study carried out in Sudan, E. coli represented the most frequently isolated bacterium among GNB, followed by
Pseudomonas aeruginosa [31]. Meanwhile, a study conducted in Egypt found that the GNB most frequently isolated from all samples was Klebsiella pneumoniae followed by E. coli [32].

Regarding GPB, CoNS represented the most frequent species isolated in our study $(32,56.1 \%)$, followed by Enterococcus faecium (10, 17.5\%), Enterococcus faecalis $(5,8.7 \%)$ and Staphylococcus aureus (4, 7.0\%). These results are comparable to the aforementioned Italian study, where CoNS were the most common species, followed by Enterococcus spp., viridans group streptococci (VGS) and Staphylococcus aureus (11). In the Indian study, the most frequent GPB isolates were CoNS, then Staphylococcus aureus, Streptococcus spp., and Enterococcus spp. (14).

In our study, the bacteria most commonly isolated were Pseudomonas aeruginosa (22\%), E. coli (16.3\%), and Staphylococcus epidermidis (11.4\%), followed by Enterococcus faecium and Staphylococcus haemolyticus $(8.1 \%$ each), and then Klebsiella pneumonia, Acinetobacter baumanii, and Staphylococcus hominis (4.9\% each). In comparison, when looking at patients with hematologic malignancies in Japan, E. coli was the most commonly seen bacterium, followed by Klebsiella spp., Pseudomonas aeruginosa, Staphylococcus aureus, Enterobacter spp. Citrobacter spp., and Acinetobacter spp. [33].

Hard to spot but lethal if missed, invasive fungal infections-predominantly caused by Aspergillus and Candida-are the leading infectious cause of mortality in patients with myelosuppression due to chemotherapy [34]. In our study, Candida had the highest share of fungal infections, in contrast to a study in Italy where most infections were caused by Aspergillus spp., followed by Candida [35].

In our study, Pseudomonas aeruginosa exhibited high resistance to ciprofloxacin (60\%), in concordance with numbers found in similar Italian studies [30, 36], and with a Spanish study that observed resistance to ciprofloxacin among cancer patients in general [37]. Pseudomonas aeruginosa isolates in our study also had high resistance to carbapenems, including imipenem (59.3\%), meropenem (48\%), and gentamicin (48\%). These numbers resemble those found in another study where the resistance rate to carbapenems was 60\% [36], and in an Italian study where the resistance rate to meropenem was $71.2 \%$ [30]. However, this is in contrast to an American study that found the resistance to imipenem seen among solid and hematological cancer patients was only $6 \%$ [38]. Also among our Pseudomonas aeruginosa isolates, piperacillin resistance was found to be $54.2 \%$, while in a previously mentioned study it was found to be $24 \%$ [36]. Meanwhile, among cephalosporins, cefepime, and ceftazidime, resistance rates were $16 \%$ and $24 \%$ respectively. The reasons 
behind these low rates of resistance to cephalosporins are the infrequent use of these agents, as the prescription of cefepime is highly restricted, and piperacillin-tazobactam is the most commonly used initial therapy for neutropenic fever instead. This highlights the importance of diversification of antibiotic use, such as prescribing thirdgeneration cephalosporins (ceftazidime) for neutropenic fever [39], to avoid selection of carbapenem resistance by extensive carbapenem use. However, the selection of empiric antimicrobial therapy should be based on multiple factors, including but not limited to the clinical status of the patient, previous cultures and colonization, and institutional antibiograms [40, 41].

Among GNB, 21 CRE (31.8\%) were detected, more than that seen in febrile neutropenic patients with hematological cancer in Japan [42]. In our study, the resistance of $E$. coli isolates to amikacin was only $5 \%$, similar to the results of another study where $85.2 \%$ of $E$. coli isolates were found to be sensitive to amikacin [30]. On the other hand, ESBL-E. coli exhibited $100 \%$ resistance to both cephalosporins and ampicillin, similar to previous research, where the vast majority of ESBL-producing isolates were resistant to all generations of cephalosporins [42]. E. coli in our study also exhibited high resistance to levofloxacin and TMP-SMX (75\% and 63.2\%, respectively), similar to the results found in a previous study [30]. This could be due to the frequent use of fluoroquinolones (especially levofloxacin) for prophylaxis in patients with prolonged neutropenia [39].

Regarding the six isolates of Acinetobacter baumannii, the highest resistance rates were observed to carbapenems (80\% to meropenem and $83.3 \%$ to imipenem) and piperacillin-tazobactam (83.3\%) similar to a related study held in Turkey [43]. Isolates also exhibited high resistance to gentamicin $(66.7 \%)$. Four of these isolates were tested for resistance to colistin and all were sensitive, in agreement with prior research where all isolates of Acinetobacter baumannii were susceptible to colistin [44]. Finally, the six isolates of Klebsiella pneumonia were $100 \%$ susceptible to piperacillin/tazobactam, carbapenems, fluoroquinolones, ceftazidime, and cefepime. In other studies, $55.8 \%$ of Klebsiella isolates were resistant to piperacillin/ tazobactam [30], 44.9\% were resistant to meropenem while $1 \%$ were resistant to imipenem [38], 69.8\% were resistant to ciprofloxacin, $58.1 \%$ were resistant to ceftazidime [30], and 20\% were resistant to cefepime [42].

Among the 10 Enterococcus faecium isolates and the 5 Enterococcus faecalis isolates, 90\% of Enterococcus faecium isolates were VRE while none of the Enterococcus faecalis isolates were VRE. Regarding Enterococcus faecium, $40 \%$ of isolates were resistant to streptomycin, $30 \%$ were resistant to gentamicin, and $11.1 \%$ were resistant to tigecycline. Meanwhile, Enterococcus faecalis species had
$80 \%$ resistance to streptomycin, $50 \%$ resistance to gentamicin, and $33.3 \%$ resistance to tigecycline. In particular, none of the Enterococcus faecalis or Enterococcus faecium isolates was resistant to linezolid, in agreement with prior research [30].

Among the CoNS (Staph. epidermidis, hominis and haemolyticus), no isolates were resistant to vancomycin or linezolid, while $93.3 \%$ were resistant to oxacillin, similar to the results of a previous study [30]. Regarding the 14 isolates of Staphylococcus epidermidis, all were resistant to penicillin and cephalosporins, and $54.5 \%$ were resistant to trimethoprim-sulfamethoxazole. Regarding the four isolates of Staphylococcus aureus, 75\% were resistant to oxacillin, a high percentage compared to patients in Italy (36.4\%) [30]. Additionally, 66.6\% were resistant to cefuroxime and $50 \%$ were resistant to ceftriaxone. However, all were sensitive to both vancomycin and linezolid, similar to those in the former Italian study [30].

Regarding antifungal resistance rates, all were sensitive to caspofungin, comparable to a similar study in which caspofungin resistance rates were 5\% [45]. All were sensitive to fluconazole, voriconazole, flucytosine, and micafungin. When reviewing the literature on Candida infections in patients with hematologic malignancies, a study showed that $27.6 \%$ [37] were resistant to fluconazole. Meanwhile, in another study, $8 \%$ of Candida were resistant to voriconazole and $5 \%$ were resistant to caspofungin [45].

$51.5 \%$ of GNB and $68.4 \%$ of GPB in this study were MDRO. Among GNB, Acinetobacter baumanni had the highest rate of MDRO (83.3\%), whereas among GPB, CoNS had the highest rate $(81.3 \%)$. Meanwhile, in a similar study in which MDROs were isolated in $13 \%$ of patients, the most frequently isolated MDRO was Klebsiella pneumoniae, followed by MRSA, Acinetobacter baumanni, Pseudomonas, E. coli, and VRE [46].

This study is the first in Palestine to determine the microbial profile of infections in patients with hematological malignancies. However, there were some limitations to our study. First, not all data were written in the patient's medical reports such as white blood cell counts, absolute neutrophil counts, and patient temperatures at the time of culture, so we could not assess neutropenic fever and its relationship with other variables. Furthermore, some data were not collected, such as the last time a patient received a chemotherapy session. Second, our data were collected from only one center that may not be representative of other centers. Third, some patients died or left the hospital before the culture results were ready, so they did not receive any treatment other than empirical antibiotics. Finally, the study did not assess increases in antibiotic resistance year over year. 


\section{Conclusions}

Patients with hematologic malignancies are at risk for a variety of serious infections that cause significant morbidity and mortality. The most common bacterial isolates among GNB were Pseudomonas aeruginosa and E. coli, while coagulase-negative staphylococci and Enterococcus faecium were the most common among GPB. Our study showed alarming rates of resistance to the most widely used antibiotics, thus highlighting the need to develop local guidelines for antimicrobial use based on local resistance patterns of these organisms. This study also emphasizes the need to develop antimicrobial stewardship programs in local hospitals. Enforcing the implementation of infection control policies would help curb the spread of these MDROs and reduce the morbidity, mortality, and economic burden of these serious infections.

\begin{abstract}
Abbreviations
GNB: Gram-negative bacteria; GPB: Gram-positive bacteria; ALL: Acute lymphoid leukemia; AML: Acute myeloid leukemia; MM: Multiple myeloma; CLL: Chronic lymphoid leukemia; E. coli: Escherichia coli; ESBL: Extended-spectrum beta-lactamase; IRB: Institutional Review Board; SD: Standard deviation; CoNS: Coagulase negative staphylococcus; VRE: Vancomycin resistance enterococcus; MDRO: Multidrug resistant organisms; spp.: Species; CRE: Carbapenemresistant Enterobacteriaceae; DTR: Difficult-to-treat resistance.
\end{abstract}

\section{Acknowledgements}

The authors thank An-Najah National University for providing an opportunity to carry out this study.

\section{Authors' contributions \\ $\mathrm{GA}, \mathrm{MZ}$ and $\mathrm{BQ}$ collected data, performed analysis and drafted the original manuscript. AA and RA provided logistical assistance, designed the study, organized, supervised, participated in the field study, and assisted in writing and the production of the final version of the manuscript. SA and AAK col- lected new data for revised version; interpreted the data, revised the manu- script for all new reviewers' concerns that have been addressed, and critically revised the draft for important intellectual content. SHZ conceptualised and designed the study; coordinated, supervised, and analysed the data; critically reviewed the manuscript; interpreted the results and assisted in writing the final manuscript. All authors read and approved the final manuscript.}

\section{Funding}

Not available.

\section{Availability of data and materials}

The data sets used and/or analysed during this study are available from the corresponding author on reasonable request.

\section{Declarations}

\section{Ethics approval and consent to participate}

The proposal was reviewed and accepted on September 22, 2019, with the permission of An-Najah National University Hospital. The approval of the Institutional Review Board (IRB) Committee of An-Najah National University was obtained on July 24, 2019 (Archived number: AN4June2019). All methods used in the study were carried out in accordance with relevant guidelines and regulations. IRB of An-Najah National University waived the need for informed consent since we used retrospective data.

\section{Consent for publication}

Not applicable.

\section{Competing interests}

The authors declare that they have no competing interests.

\section{Author details}

${ }^{1}$ Department of Medicine, College of Medicine and Health Sciences, An-Najah National University, Nablus 44839, Palestine. ${ }^{2}$ Department of Biomedical Sciences, Faculty of Medicine and Health Sciences, An-Najah National University, Nablus 44839, Palestine. ${ }^{3}$ Department of Pathology, An-Najah National University Hospital, Nablus 44839, Palestine. ${ }^{4}$ Department of Hematology and Oncology, An-Najah National University Hospital, Nablus 44839, Palestine. ${ }^{5}$ Department of Clinical and Community Pharmacy, College of Medicine and Health Sciences, An-Najah National University, Nablus 44839, Palestine. ${ }^{6}$ Division of Clinical Pharmacy, Department of Hematology and Oncology, An-Najah National University Hospital, Nablus 44839, Palestine. ${ }^{7}$ Poison Control and Drug Information Center (PCDIC), College of Medicine and Health Sciences, An-Najah National University, Nablus 44839, Palestine. ${ }^{8} \mathrm{Clini}-$ cal Research Center, An-Najah National University Hospital, Nablus 44839, Palestine. ${ }^{9}$ Department of Pharmacy, College of Medicine and Health Sciences, An-Najah National University, Nablus 44839, Palestine.

Received: 30 July 2020 Accepted: 31 January 2022

Published online: 10 February 2022

\section{References}

1. Legese $\mathrm{MH}$, Weldearegay GM, Asrat D. Extended-spectrum betalactamase- and carbapenemase-producing Enterobacteriaceae among Ethiopian children. Infect Drug Resist. 2017;10:27-34.

2. Blijlevens NM, Donnelly JP, de Pauw BE. Microbiologic consequences of new approaches to managing hematologic malignancies. Rev Clin Exp Hematol. 2005;9(2):E2.

3. Zembower TR. Epidemiology of infections in cancer patients. Cancer Treat Res. 2014;161:43-89.

4. Zembower T. Epidemiology of infectious complications in cancer patients. Cancer Treat Res. 1998;96:33-75.

5. Gedik H, Simşek F, Kantürk A, Yildirmak T, Arica D, Aydin D, Demirel N, Yokuş O. Bloodstream infections in patients with hematological malignancies: which is more fatal_cancer or resistant pathogens? Ther Clin Risk Manag. 2014;10:743-52.

6. Montassier E, Batard E, Gastinne T, Potel G, de La Cochetière MF. Recent changes in bacteremia in patients with cancer: a systematic review of epidemiology and antibiotic resistance. Eur J Clin Microbiol Infect Dis. 2013;32(7):841-50.

7. Islas-Munoz B, Volkow-Fernandez P, Ibanes-Gutierrez C, VillamarRamirez A, Vilar-Compte D, Cornejo-Juarez P. Bloodstream infections in cancer patients. Risk factors associated with mortality. Int J Infect Dis. 2018;71:59-64.

8. Blennow $\mathrm{O}$, Ljungman $\mathrm{P}$. The challenge of antibiotic resistance in haematology patients. Br J Haematol. 2016;172(4):497-511.

9. Walwyn M, Nicholson A, Lee MG, Wharfe G, Frankson MA. Febrile neutropaenia in cancer patients. West Indian Med J. 2010;59(2):209-14.

10. Fentie A, Wondimeneh Y, Balcha A, Amsalu A, Adankie BT. Bacterial profile, antibiotic resistance pattern and associated factors among cancer patients at University of Gondar Hospital, Northwest Ethiopia. Infect Drug Resist. 2018;11:2169-78.

11. Husseini A, Abu-Rmeileh NM, Mikki N, Ramahi TM, Ghosh HA, Barghuthi N, Khalili M, Bjertness E, Holmboe-Ottesen G, Jervell J. Cardiovascular diseases, diabetes mellitus, and cancer in the occupied Palestinian territory. Lancet. 2009;373(9668):1041-9.

12. Halahleh K, Gale RP. Cancer care in the Palestinian territories. Lancet Oncol. 2018;19(7):e359-64.

13. El-Astal Z. Bacterial pathogens and their antimicrobial susceptibility in Gaza Strip, Palestine. Pak J Med Sci. 2004;20(4):365-70.

14. Sweileh WM. Global research activity on antimicrobial resistance in foodproducing animals. Arch Public Health. 2021;79(1):49.

15. Sweileh WM. Bibliometric analysis of peer-reviewed literature on antimicrobial stewardship from 1990 to 2019. Global Health. 2021;17(1):1.

16. Sweileh WM, AbuTaha AS, Sawalha AF, Al-Khalil S, Al-Jabi SW, Zyoud SH. Bibliometric analysis of worldwide publications on multi-, extensively, 
and totally drug-resistant tuberculosis (2006-2015). Multidiscip Respir Med. 2016;11:45.

17. Sweileh WM, Al-Jabi SW, Sawalha AF, AbuTaha AS, Zyoud SH. Bibliometric analysis of publications on Campylobacter: (2000-2015). J Health Popul Nutr. 2016;35(1):39.

18. Sweileh WM, Al-Jabi SW, Zyoud SH, Sawalha AF, Abu-Taha AS. Global research output in antimicrobial resistance among uropathogens: a bibliometric analysis (2002-2016). J Glob Antimicrob Resist. 2018;13:104-14.

19. Sweileh WM, Sawalha AF, Al-Jabi S, Zyoud SH. Bibliometric analysis of literature on antifungal triazole resistance: 1980-2015. Germs. 2017;7(1):19-27.

20. Sweileh WM, Shraim NY, Al-Jabi SW, Sawalha AF, AbuTaha AS, Zyoud SH. Bibliometric analysis of global scientific research on carbapenem resistance (1986-2015). Ann Clin Microbiol Antimicrob. 2016;15(1):56.

21. Lim C, Takahashi E, Hongsuwan M, Wuthiekanun V, Thamlikitkul V, Hinjoy S, Day NP, Peacock SJ, Limmathurotsakul D. Epidemiology and burden of multidrug-resistant bacterial infection in a developing country. Elife. 2016; 5 .

22. Medina E, Pieper DH. Tackling threats and future problems of multidrugresistant bacteria. Curr Top Microbiol Immunol. 2016;398:3-33.

23. An-najah National University Hospital. https://nnuh.org/ar/mn-nhn/nshImstshf/. Accessed 9 Oct 2021.

24. Magiorakos AP, Srinivasan A, Carey RB, Carmeli Y, Falagas ME, Giske CG, Harbarth S, Hindler JF, Kahlmeter G, Olsson-Liljequist B, et al. Multidrugresistant, extensively drug-resistant and pandrug-resistant bacteria: an international expert proposal for interim standard definitions for acquired resistance. Clin Microbiol Infect. 2012;18(3):268-81.

25. Kadri SS, Adjemian J, Lai YL, Spaulding AB, Ricotta E, Prevots DR, Palmore TN, Rhee C, Klompas M, Dekker JP, et al. Difficult-to-treat resistance in gram-negative bacteremia at 173 US hospitals: retrospective cohort analysis of prevalence, predictors, and outcome of resistance to all firstline agents. Clin Infect Dis. 2018;67(12):1803-14.

26. Dardiotis E, Aloizou AM, Markoula S, Siokas V, Tsarouhas K, Tzanakakis G, Libra M, Kyritsis AP, Brotis AG, Aschner M, et al. Cancer-associated stroke: pathophysiology, detection and management (Review). Int J Oncol. 2019;54(3):779-96.

27. Alibek K, Bekmurzayeva A, Mussabekova A, Sultankulov B. Using antimicrobial adjuvant therapy in cancer treatment: a review. Infect Agent Cancer. 2012;7(1):33.

28. Perez F, Adachi J, Bonomo RA. Antibiotic-resistant gram-negative bacterial infections in patients with cancer. Clin Infect Dis. 2014;59(Suppl 5):S335-339.

29. Saghir S, Faiz M, Saleem M, Younus A, Aziz H. Characterization and anti-microbial susceptibility of gram-negative bacteria isolated from bloodstream infections of cancer patients on chemotherapy in Pakistan. Indian J Med Microbiol. 2009;27(4):341-7.

30. Trecarichi EM, Pagano L, Candoni A, Pastore D, Cattaneo C, Fanci R, Nosari A, Caira M, Spadea A, Busca A, et al. Current epidemiology and antimicrobial resistance data for bacterial bloodstream infections in patients with hematologic malignancies: an Italian multicentre prospective survey. Clin Microbiol Infect. 2015;21(4):337-43.

31. Nurain AM, Bilal NE, Ibrahim ME. The frequency and antimicrobial resistance patterns of nosocomial pathogens recovered from cancer patients and hospital environments. Asian Pac J Trop Biomed. 2015;5(12):1055-9.

32. Ashour HM, El-Sharif A. Species distribution and antimicrobial susceptibility of gram-negative aerobic bacteria in hospitalized cancer patients. J Transl Med. 2009;7:14

33. Mimura W, Fukuda H, Akazawa M. Antimicrobial utilization and antimicrobial resistance in patients with haematological malignancies in Japan: a multi-centre cross-sectional study. Ann Clin Microbiol Antimicrob. 2020;19(1):7.

34. Badiee $P$, Hashemizadeh Z. Opportunistic invasive fungal infections: diagnosis and clinical management. Indian J Med Res. 2014;139(2):195-204.

35. Pagano L, Caira M, Candoni A, Offidani M, Fianchi L, Martino B, Pastore D, Picardi M, Bonini A, Chierichini A, et al. The epidemiology of fungal infections in patients with hematologic malignancies: the SEIFEM-2004 study. Haematologica. 2006;91(8):1068-75.

36. Trecarichi EM, Tumbarello M, Caira M, Candoni A, Cattaneo C, Pastore D, Fanci R, Nosari A, Vianelli N, Busca A, et al. Multidrug resistant Pseudomonas aeruginosa bloodstream infection in adult patients with hematologic malignancies. Haematologica. 2011;96(1):e1-3; author reply e4.

37. Puig-Asensio M, Ruiz-Camps I, Fernandez-Ruiz M, Aguado JM, Munoz P, Valerio M, Delgado-Iribarren A, Merino P, Bereciartua E, Fortun J, et al. Epidemiology and outcome of candidaemia in patients with oncological and haematological malignancies: results from a population-based surveillance in Spain. Clin Microbiol Infect. 2015;21(5):491 e491-410.

38. Wisplinghoff $\mathrm{H}$, Seifert $\mathrm{H}$, Wenzel RP, Edmond MB. Current trends in the epidemiology of nosocomial bloodstream infections in patients with hematological malignancies and solid neoplasms in hospitals in the United States. Clin Infect Dis. 2003;36(9):1103-10.

39. Lee DG, Kim SH, Kim SY, Kim CJ, Park WB, Song YG, Choi JH. Evidencebased guidelines for empirical therapy of neutropenic fever in Korea. Korean J Intern Med. 2011;26(2):220-52.

40. Tam CS, O'Reilly M, Andresen D, Lingaratnam S, Kelly A, Burbury K, Turnidge J, Slavin MA, Worth LJ, Dawson L, et al. Use of empiric antimicrobial therapy in neutropenic fever. Internal Med J. 2011;41(1b):90-101.

41. Zimmer AJ, Freifeld AG. Optimal management of neutropenic fever in patients with cancer. J Oncol Pract. 2019;15(1):19-24.

42. Chong Y, Yakushiji H, Ito Y, Kamimura T. Cefepime-resistant Gram-negative bacteremia in febrile neutropenic patients with hematological malignancies. Int J Infect Dis. 2010;14(Suppl 3):e171-175.

43. Kara O, Zarakolu P, Ascioglu S, Etgul S, Uz B, BuyukasikY, Akova M. Epidemiology and emerging resistance in bacterial bloodstream infections in patients with hematologic malignancies. Infect Dis (Lond). 2015;47(10):686-93.

44. Nogbou ND, Phofa DT, Nchabeleng M, Musyoki AM. Investigating multidrug resistant Acinetobacter baumannii isolates at a tertiary hospital in Pretoria South Africa. Indian J Med Microbiol. 2021;39(2):218-23.

45. Sipsas NV, Lewis RE, Tarrand J, Hachem R, Rolston KV, Raad II, Kontoyiannis DP. Candidemia in patients with hematologic malignancies in the era of new antifungal agents (2001-2007). Cancer. 2009;115(20):4745-52.

46. Perdikouri EIA, Arvaniti K, Lathyris D, Apostolidou Kiouti F, Siskou E, Haidich AB, Papandreou C. infections due to multidrug-resistant bacteria in oncological patients: insights from a five-year epidemiological and clinical analysis. Microorganisms. 2019;7(9):277.

\section{Publisher's Note}

Springer Nature remains neutral with regard to jurisdictional claims in published maps and institutional affiliations.

Ready to submit your research? Choose BMC and benefit from

- fast, convenient online submission

- thorough peer review by experienced researchers in your field

- rapid publication on acceptance

- support for research data, including large and complex data types

- gold Open Access which fosters wider collaboration and increased citations

- maximum visibility for your research: over 100M website views per year

At BMC, research is always in progress.

Learn more biomedcentral.com/submissions 